\title{
Research on Interior Ballistic of a Gas Multi-stage Cylinder Ejection
}

\author{
Mei Huang ${ }^{1, a}$, Yi Jiang ${ }^{2}$ and Boman Wang ${ }^{1}$ \\ ${ }^{1}$ School of Aerospace Engineering, Beijing Institute of Technology, 100081, Beijing, China
}

\begin{abstract}
Aimed at increasing aircraft's leaving speed based on a determinate length of aircraft container and ensuring the aircraft's acceleration below one certain value, a new gas multistage cylinder ejection is proposed in this paper, and at the same time the primary design and the interior ballistic evaluating are processed. The simulation results can be used for the design of gas-catapult device and provide theoretical support for parameter optimizations in trajector.
\end{abstract}

\section{Introduction}

According to the launching power, the launching method for aircraft can be divided into self-launch and ejection. Ejection is a way that uses external power to catapult the aircraft out of the launching tube. When the aircraft reaches to a predetermined height, the engine of the aircraft is ignited and it flies as programmed[1]. Compared with the other launching technology, ejection has its unique advantages. Ejection can make the aircraft get a certain initial speed, thus achieving a longer range. Besides, the aircraft is installed in a sealed launching tube. And thus it can provide a stable storage conditions with constant temperature and pressure. So it has been widely used and developed since the late 1950s. Ejection can be classified into compressed air ejection, gas ejection and gas-steam ejection by working medium. In form of launching device, it can be divided into tube ejection and piston cylinder ejection too[2].

The gas multi-stage cylinder ejection proposed in this paper is built on the basis of piston cylinder ejection. Because of the latter's structure limitations, its actual stroke is less than half the length of the launching tube.If we want to increase the aircraft's leaving velocity, it can be solved by lengthening the launching tube or by increasing the mass of propellants. However, the former will affect the maneuverability and traffic-ability characteristic of the launching vehicle. And the latter will lead to the increase of aircraft's overload[3]. In view of the above contradiction, a new gas multi-stage cylinder ejection is put forward in this paper. The gas generator (also called HPC which is short for high-pressure chamber) is used as the power source of the ejection to gurantee the output power. The design of multi-stage cylinder can not only increase the ejection stroke to improve the maximum ejection speed, but also prevent the aircraft from ablation[4-5]. At last, the the interior ballistic performances of this ejection are calculated with numerical method and verified by dynamic analysis.

\section{Physical model}

\footnotetext{
${ }^{\mathrm{a}}$ Corresponding author : 18612786701@163.com
} 
In the design of the ejection device, the gas generator and the multi-stage cylinders are integrated to simplify the structure and ensure the balance of multi-stage actuating cylinders. The ejection device, as shown in the Fig. 1, is mainly composed of four parts: the gas generator and the LPC(which is short for low-pressure chamber), four groups of multi-stage cylinders and the shell of the device. And each group is composed of ten stage cylinders, each stage has an effective stroke of $1200 \mathrm{~mm}$.

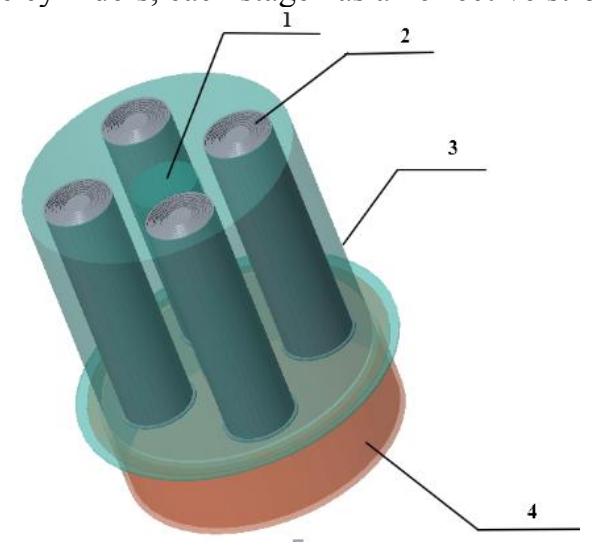

Figure 1. Structure of the gas multi-stage cylinder ejection:

1-the gas generator; 2-multi-stage cylinder; 3-shell; 4-the LPC

\section{Mathematical model}

\subsection{The motion rule of missile}

During the ejection process, the missile moves upward along the catapult. It has four forces in the axial direction, namely, ejection force, gravity, and friction. The acceleration expression of missile motion can be obtained from Newton's second law.

$$
m \frac{d v_{m}}{d t}=F_{e}-F_{f}-F_{g}
$$

where, $F_{e}$ is the ejection force, $F_{f}$ is the friction force, $F_{g}$ is the gravity.

\subsection{Zero dimensional interior ballistic hypothesis}

- The gas in high-pressure chamber is treated as perfect gas. And it ingredients, physical and chemical properties will not vary.

- The gas in the low-pressure chamber is treated as perfect gas. And it follows the ideal gas equation.

- The spatial distribution of pressure and temperature in low-pressure chamber is not considered. And suppose they are the same at the time.

- The heat exchange from the chamber wall of the high-pressure chamber to the gas in the lowpressure chamber is neglected.

\subsection{Zero dimensional interior ballistics Equations}




$$
\left\{\begin{array}{l}
\frac{d P_{1}}{d t}=\frac{R T_{1}}{V_{1}}\left(\dot{m}_{b}-\dot{m}_{t 1}\right) \\
\frac{d e}{d t}=u \\
\frac{d v_{m}}{d t}=\frac{S_{2} P_{2}}{\varphi m} \\
\frac{d l_{m}}{d t}=v_{m} \\
\dot{m}_{b}=\rho_{p} S_{b} u \\
\dot{m}_{t 1}=\varphi_{21} K_{0} \frac{P_{1} S_{t 1}}{\sqrt{R T_{1}}} \\
u=a p_{1}^{n} \\
P_{2}=\frac{m_{t 1} R T_{2}}{V_{20}+S_{2} l_{m}} \\
T_{2}=\chi_{2} k T_{1}-\frac{(k-1) S_{2} P_{2} v_{m}}{R \dot{m}_{t 1}}
\end{array}\right.
$$

Where, $P_{1}$ is the pressure in HPC; $T_{1}$ is the temperature in HPC; $\dot{m}_{b}$ is the burning rate of propellant; $\dot{m}_{t 1}$ is the mass flow; $v_{m}$ is the velocity of missile; $\rho_{p}$ is the density of the propellant; $S_{b}$ is the area of the burning face; $\varphi_{21}$ is the flux modificative coefficient of HPC; $S_{t 1}$ is the area of the throat at the nozzle; $n$ is the gas index of pressure; $k$ is the specific heat ratio, and $K_{0}$ is a constant connecting with $k, K_{0}=\sqrt{k}\left(\frac{2}{k+1}\right)^{\frac{k+1}{2(k-1)}}[6]$.

\subsection{Algorithm realization of interior ballistic equations}

Matlab is used to solve the above equations. It has the characteristics of small code, high programming efficiency, powerful function, and easy to use. On the other hand, it has a unique advantage especially in the numerical calculation and visualization.

The algorithm realization of interior ballistic equations is as follows: Firstly, the above equations can be written into a set of ordinary differential equations. Then, code the program adopting the Runge-Kutta method. At last, Matlab is used to solve the initial value problem of ordinary differential equations.

\section{Simulation and analysis}

\subsection{Calculation conditions}

Before the simulation analysis of the multi-stage ejection device, some calculation conditions should be given first. 
According to the reference[7-8], the $P_{1}-t$ curve and the nozzle are designed shown in Fig. 2-3. Besides, the design performance index is given in Table. 1.

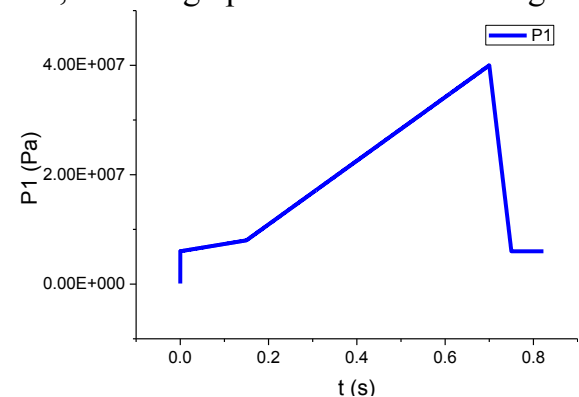

Figure 2. Pressure curve of the HPC .

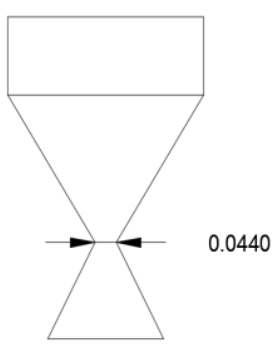

Figure 3. Structure of the nozzle.

Table 1. Design performance index.

\begin{tabular}{cc}
\hline Performance index & Value \\
\hline Leaving velocity & $\geq 25 \mathrm{~m} / \mathrm{s}$ \\
Overload & $\leq 8 \mathrm{~g}$ \\
Leaving time & $\leq 1 \mathrm{~s}$ \\
\hline
\end{tabular}

\subsection{Internal ballistic simulation}

Using the four order Runge-Kutta method to solve the ballistic equations combined with the above scheme design, the corresponding curves of the interior ballistic parameters varying with time can be obtained. And they are illustrated in Fig. 4-7.

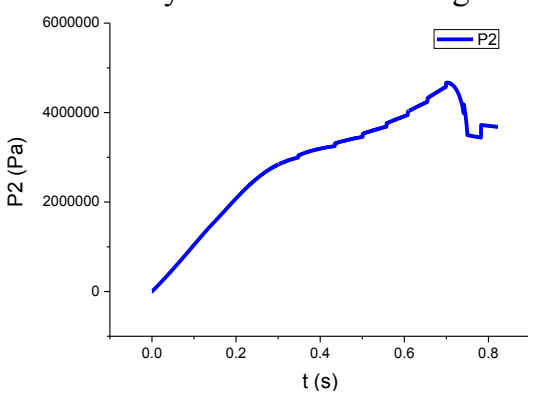

Figure 4. Pressure curve of the LPC.

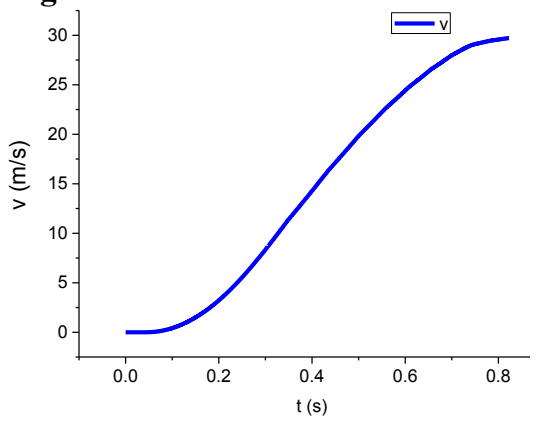

Figure 6. Velocity curve of the payload.

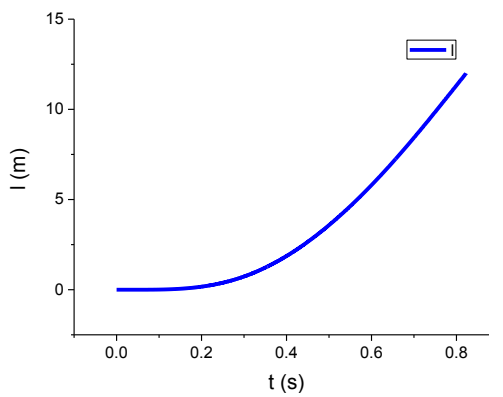

Figure 5. Displacement curve of the payload.

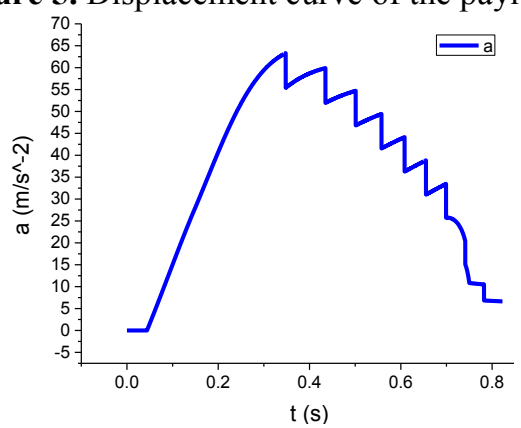

Figure 7. Acceleration curve of the payload.

In Fig. 4, due to the fact that the contact area between the multi-stage cylinder and the payload is gradually decreasing with the full extension of each stage, the pressure in the LPC is on the rise, and 
the maximum pressure appears at about $0.8 \mathrm{~s}$ and the peak value is about $5.5 \mathrm{MPa}$. As the decrease of the contact area is faster than the increase of the pressure in the LPC, the overall acceleration of the payload tends to decrease. So from Fig. 7, it can be seen that the acceleration of the payload declines after a certain stage cylinder is fully extended.

From the above figures, the total time of payload ejection is about $0.83 \mathrm{~s}$. In Fig. 6 , the leaving velocity of the payload is about $30 \mathrm{~m} / \mathrm{s}$. It can be seen from Fig. 7 that the maximum acceleration of the payload occurs at about $0.3 \mathrm{~s}$ and it is less than $6 \mathrm{~g}$. In brief, the three meet the design requirements.

\subsection{Dynamics analysis}

As is shown in Fig. 8, the dynamics analysis simulation model is built in ADAMS. In the dynamics simulation, it is supposed that the motion between every stage cylinder is linear relatively to the axis of the cylinder. In addition, the payload is only subjected to the contact force at the bottom while the radial direction is unconstrained. And friction is small and can be neglected.Among them, the cylinder collision coefficient is set as steel to steel shown in Table. 2.

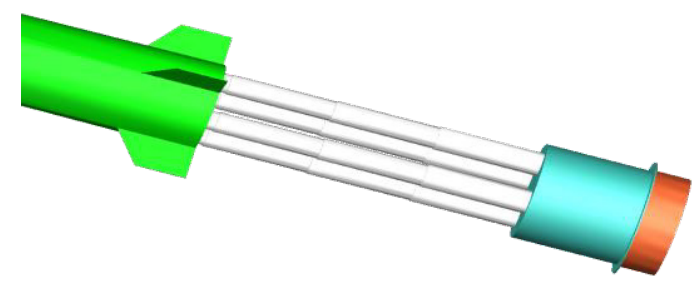

Figure 8. Dynamic model.

Table 2. The cylinder collision coefficient.

\begin{tabular}{c|c}
\hline Parameter & Value \\
\hline impact stiffness & $1.0 \mathrm{e} 5 \mathrm{~N} / \mathrm{mm}$ \\
damping & $50 \mathrm{Ns} / \mathrm{mm}$ \\
index & 1.5 \\
depth & $0.1 \mathrm{~mm}$ \\
\hline
\end{tabular}

Combined with zero dimensional interior ballistic curve obtained before, the results of dynamics simulation are shown in Fig. 9-12.

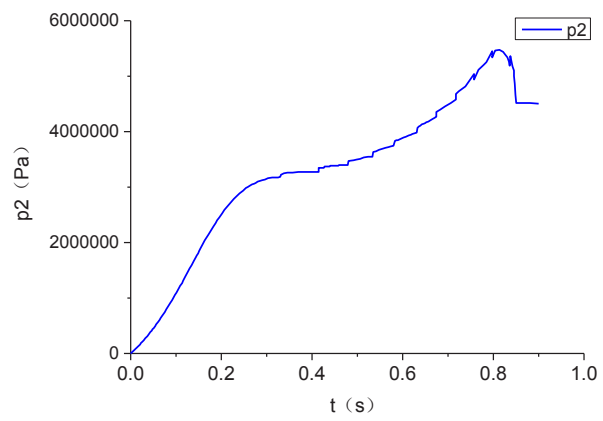

Figure 9. Pressure curve of the LPC .

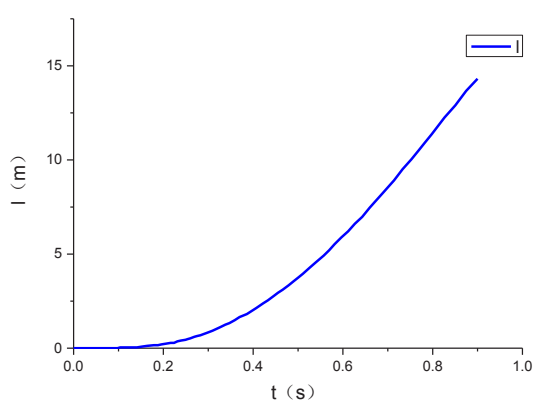

Figure 10. Displacement curve of the payload. 


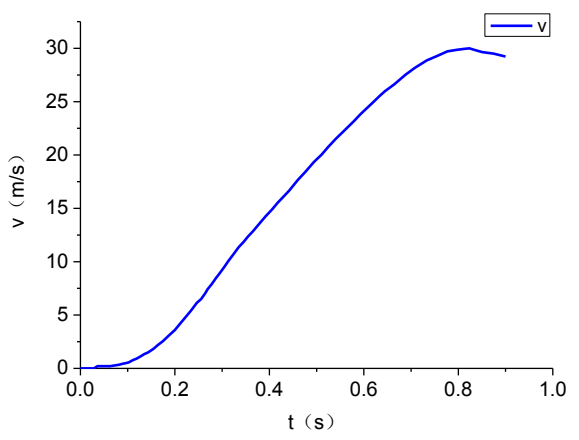

Figure 11. Velocity curve of the payload.

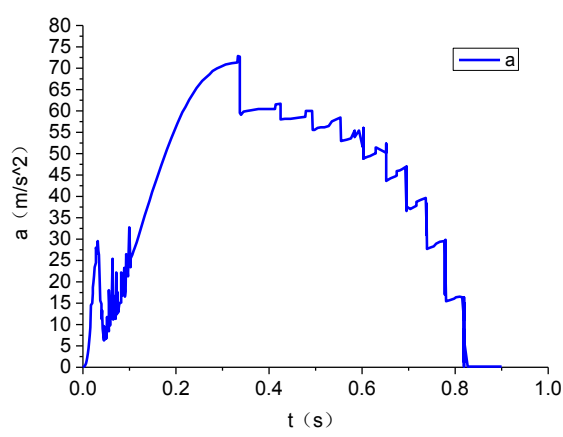

Figure 12. Acceleration curve of the payload.

As shown in Fig. 10, it takes $0.81 \mathrm{~s}$ to leave the launching tube for the payload and its displacement is $11.8 \mathrm{~m}$. It can be seen from Fig. 11 that the velocity of the payload increases smoothly and reaches the maximum at the end of the ejection phase with a speed of $29.7 \mathrm{~m} / \mathrm{s}$. The maximum overload of the payload is about $7.3 \mathrm{~g}$, which appears at the end of the first stage and then decreases progressively shown in Fig. 12.

From the above analysis, the gas multi-cylinder ejection proposed in this paper, without changing the length and diameter of the launching tube, which increases the leaving speed while maintaining a low overload, achieves the design requirements.

\section{Conclusion}

In the ejection process for payload, high leaving velocity and low overload are contradicted. In view of this problem, a new gas multi-stage cylinder ejection is proposed in this paper. Through preliminary design, interior ballistic calculation and dynamic analysis, it is shown that the ejection can achieve the expected goals to solve the problem in a certain extent. Furthermore, it provides the valuable reference and inspiration to further optimization for new ejections.

\section{References}

1. S.Z. Rui and Y.M. Xing. Development Rearch on Aircraft Launching Power system. T. M. T. 5, 4-9 (2009)

2. R. Ren, D.W. Ma, L. Yao, Z. Liu and Q. He. Theoretical Modeling and Performance Research on Multi-stage Pneumatic and Hydraulic Catapult Device. A. A 37, 1365-72 (2016)

3. F. Yan, S.Y. Shi and Y. Jiang. Rearch on Interior Ballistic of Gas-steam Ejection. S. S. A. T 39, 118-22 (2017)

4. S.Z. Rui and Y.M. Xing. Comparative Studies of Interial Ballistic Performance among Several aircraft Eject Power System. J. B. U. A. A 35, 766-770 (2009)

5. P.Y. Bai, J. Qiao. Analysis about the Interior Trajectory of Two-Step Cylinder Ejection Device. M. D. T 35,44-49 (2007)

6. D.C. Tan 2015 Interior Ballistics for Ejection (Beijing: Beijing Institute of Technology Press)

7. F.B. Yang, D.W. Ma, F. Yang, et al. Interior ballistics modeling and Calculation of high-pressure ejection device. A. A 34, 527-534 (2013)

8. R.G. Jiang. Aircraft Ejection and Design of Gas Generator. J. M. D. T 26, 9-16 (1998) 